\title{
Technology optimization study on high fragrance cold solubility instant powder of Hubei Qingzhuan tea produced by freeze-drying method
}

\author{
Hui-hui Li ${ }^{1}$, Qing-qing Xie ${ }^{1}$, Qing-mei Pan ${ }^{2}$, Si-yun He ${ }^{1}$, and Hong-wei Sui1 ${ }^{1, *}$ \\ ${ }^{1}$ College of Food Technology, Wuhan Business University, Wuhan, China \\ ${ }^{2}$ School of Food Science and Engineering, Wuhan Polytechnic University, Wuhan, China
}

\begin{abstract}
Although Chinese instant tea powder production ranks first in the world, instant dark tea powder was rare in the market. Zhaoliqiao, Changshengchuan and Yangloudong Qingzhuan tea were used to optimize the technology process of high fragrance cold solubility instant powder while absorbance value was taken as detection index by single factor experiments combined with orthogonal experiments, which could provide a beneficial reference for deep utilization of Hubei Qingzhuan tea. The data showed the particle size of tea, tea-water ratio and extracting temperature significantly affected the extraction efficiency while extraction time did little. The extraction efficiency was the highest when using 120-mesh dark tea, extracting with 1:40 tea-water ratio, and extracting temperature of $100^{\circ} \mathrm{C}$.
\end{abstract}

\section{1 introduction}

Hubei Qingzhuan tea originated in the ancient town of Yangloudong, Zhaoliqiao, Chibi City, Hubei Province, shaped like bricks, belongs to dark tea. At present, the products are mainly sold to the northwest frontier areas and Mongolia, Russia, Britain and other countries. In recent years, the tea industry in Hubei province continues to expand, especially Qingzhuan tea. In 2015, Hubei Qingzhuan tea accounted for $35 \%$ of the national dark tea market[1]. Current research shows that Qingzhuan tea also has many physiological functions such as weight loss, anti-oxidation and anti-bacteria, and has great developing value[2-4].

Instant tea powder was a solid beverage in the form of crystal, powder or flake that can be dissolved in water without tea residue, which was made of finished tea, semi-finished tea and tea by-products. At present, the production of instant tea powder in China has become the first in the world, with the annual production exceeding 20,000 tons[1]. Among them, the yield of instant black tea was the largest, followed by instant green tea, dark tea instant powder almost no. At the same time, the traditional instant tea powder had a bitter taste, light flavor, distorted color and poor cold solubility[5]. Because of rich in containing, Hubei Qingzhuan tea formed tea cream after cooling. The aroma of Qingzhuan tea was pure, which not only had the "aged fragrance", but also had the unique "woody aroma"[6]. So it was of great practical and economic value to produce "high fragrance and cold solubility" Qingzhuan tea instant powder.
The processing technology of instant tea powder mainly included extraction, filtration, sterilization and drying. The efficiency of ultrasonic assisted extraction was much higher while microwave-assisted extraction took a shorter time and was convenient for industrial production. The aroma quality of tea powder was better by using ultra-high temperature instantaneous sterilization and fragrance extraction recovery technology. At present, spray drying and freeze drying are commonly used in instant tea powder production. The speed of spray drying was faster and convenient for continuous production while freeze drying made the product better quality for the reason that more the fragrance and active ingredients were retained. Processing technology of freeze drying was researched in black and green tea instant powder[7]. In this paper ultrasonic assisted extraction and freeze-drying technology were adopted to optimize the production process of Hubei Qingzhuan tea high fragrance cold solubility instant powder by single factor experiments combined with orthogonal experiments, which could provide beneficial reference for deep utilization of Hubei Qingzhuan tea.

\section{Materials and methods}

\subsection{Materials and instruments}

Main materials: Zhaoliqiao (ZLQ), produced in Yangloudong Tea Factory, Chibi City, Hubei Province; Yangloudong (YLD), produced in Hubei Chibi Yangloudong Cultural and Ecological Industry Garden; Changshengchuan (CSC), produced in Yichang City, Hubei Province.

Main instruments: YP30002 electronic balance, 
produced by Shanghai Yueping Technology Instrument Co., Ltd.; JJ-2 tissue masher, produced by Jintan Yichen Instrument Manufacturing Co., Ltd.; standard sieve (20, 40, 60, 80, $120 \mathrm{mesh}$ ), produced by Zhejiang Shangyu Daoxu Zhangxing Yarn Screen Factory; digital display constant temperature water bath HH-S4, produced by Jintan City Jarrell Electric Co., Ltd.; TDZ4-WS desktop low speed centrifuge, produced by Hunan Xiangyi Laboratory Instrument Development Co., Ltd.; UV-5500PC ultraviolet-visible spectrophotometer, produced by Shanghai Yuan Analysis Instrument Co., Ltd.; 722 visible spectrophotometer, produced by Shanghai Youke Instrument Co., Ltd.; KX-1730QT ultrasonic instrument, produced by Beijing Kexin Instrument Co., Ltd.; freeze dryer, produced by Ningbo Yinzhou Shuangjia Instrument Co., Ltd.

\subsection{Experiment methods}

\subsubsection{Production technology and extraction test index of Hubei Qingzhuan instant tea powder}

The production process of instant tea powder used in this paper was as follows: tea raw material $\rightarrow$ ultrasonic extraction $\rightarrow$ filtration $\rightarrow$ constant volume $\rightarrow$ freeze drying. After quantitatively weighing the Qingzhuan tea, the tea leaves were crushed in a tissue crusher and sieved. Then added the tea powder to distilled water under the specified temperature at the tea-water ratio, and placed in an ultrasonic instrument equipped with water at the same temperature for ultrasonic assisted extraction. Took it out and cooled down, then centrifuged it at $4000 \mathrm{r} / \mathrm{min}$ for 10 min, and put the supernatant in a freeze dryer after dilute the solution to volume.

In order to obtain high fragrance tea powder, freeze-drying was selected in this experiment. According to the reference[7] and the actual situation, the freeze-drying conditions were as follows: first pre-freeze the dark tea extract in a $-40^{\circ} \mathrm{C}$ refrigerator, and the thickness of the extract was $10 \mathrm{~mm}$. Next we transferred the frozen tea extract to a freeze dryer, and set the freeze-drying time to $8.5 \mathrm{~h}$ and the pressure to 0.06 $\mathrm{mgHg}$.

In this experiment, the absorbance value of the dark tea extract after constant volume process was used as an index of extraction efficiency. According to the Beer-Lambert law of the spectrophotometer, when the incident light intensity, molar absorptivity coefficient remain unchanged, there was a linear relationship between the absorbance and concentration of the solution within a certain range. Therefore, the higher the absorbance value was, the more extracts in the tea powder extracted, the higher the extraction efficiency was.

\subsubsection{Full-wavelength scan of Qingzhuan tea extract and optimal incident wavelength}

After diluting the dark tea extract appropriately, it was perform a full wavelength scan using a ultraviolet-visible (UV) spectrophotometer. Then selected the appropriate wavelength as the incident wavelength.

\subsubsection{Single factor experiments of ultrasonic extraction process of Qingzhuan instant tea powder}

In this paper, the effects of different level of "particle size of tea, tea-water ratio, extracting temperature, extracting time" on extraction efficient were investigated in Qingzhuan tea instant powder. In the single-factor experiment of "tea particle size", tea leaf were crushed in a tissue crusher at different time, and finally prepared 20, $40,60,80$, and 120 mesh tea powder. All tea powder was added to water at the ratio of 1: $30(\mathrm{v} / \mathrm{v})$, and the supernatants were taken after leaching at $75^{\circ} \mathrm{C}$ for 15 minutes to measure the absorbance value. In the single factor test of "tea-water ratio", after equal-weight 60-mesh tea powder taken, different amounts of distilled water were added at the tea-water ratio of 1:10, 1:20, $1: 30,1: 40$ and 1:50. After extraction, the supernatant was diluted to constant volume, then measured the absorbance value. In the "extracting temperature" experiment, 60 mesh tea powder was added with water at a tea-water ratio of 1:30. The supernatant was taken at 0 , $25,75,90$ and $100^{\circ} \mathrm{C}$ for 15 minutes, and the absorbance value was measured. In the experiment of "extracting time", 60 mesh tea were added with water at the of 1:30 and extracted at $75^{\circ} \mathrm{C}$ for $5,10,15,20$ and 25 minutes individually, then measured the absorbance value. In this experiment, three kinds of Hubei Qingzhuan tea, including ZLQ, CSC and YLD, were used to make instant tea powder. At the same time, three parallel experiments were carried out of each tea.

\subsubsection{Orthogonal experiment of ultrasonic extraction process of Qingzhuan instant tea powder}

Based on the extraction efficiency of instant tea powder in single factor experiments, the four factors of "A: particle size of tea, B: tea-water ratio, C: extracting temperature, D: extracting time" and their three levels were selected as shown in Table 1. Nine sets of orthogonal experiment were conducted and each set was carried out three times in parallel. Orthogonal experimental samples not only needed to be measured the absorbance value, but also calculated the final yield of instant tea powder. Since the water content in the tea powder was still different after freeze-drying, the tea powder yield was just used as a reference for the extraction efficiency.

The absorbance values (Abs.) of nine sets of orthogonal experiment were calculated based on the average of three parallel experiments, and the range analysis was performed by Orthogonal Assistant II (V3.1). Variance analysis used the "univariate analysis" in the "general linear model" of SPSS19.0. The model settings did not consider interactions, but examined the main effects of various factors. 
Table 1. Factors and levels table of orthogonal experiment

\begin{tabular}{|c|c|c|c|c|}
\hline & A & B & C & D \\
\hline 1 & 60 & $1: 40$ & 75 & 5 \\
\hline 2 & 80 & $1: 30$ & 90 & 10 \\
\hline 3 & 120 & $1: 20$ & 100 & 15 \\
\hline
\end{tabular}

Note: A: particle size of tea (mesh), B: tea-water ratio, C: extracting temperature $\left({ }^{\circ} \mathrm{C}\right)$, D: extracting time $(\mathrm{min})$.

\subsubsection{Yield of instant powder of Qingzhuan tea}

Yield=(the weight of instant tea powder / the weight of tea raw materials ) $\times 100 \%$

\section{Results and analysis}

\subsection{Full-wavelength scanning of tea extract}

The full-wavelength scanning of Qingzhuan tea aqueous extract was performed in the range of 200-600 nm. The scan results are shown in Fig.1. It showed that in the range of 320-380 nm, the absorbance of Qingzhuan tea extract was higher. Taking into account the stability of the instrument and other factors, we set the optimal incident wavelength to $360 \mathrm{~nm}$. In the subsequent experiments, the absorbance value of the extract was measured in $360 \mathrm{~nm}$.

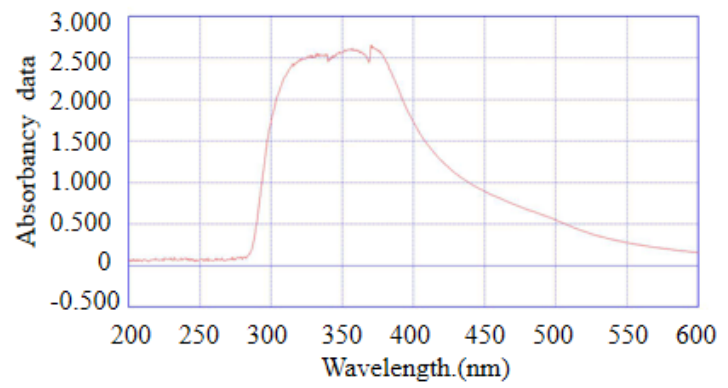

Fig.1. Full wavelength scanning of Qingzhuan tea extract

\subsection{Single factor experiments of Qingzhuan instant tea powder production technology}

\subsubsection{The effect of tea particle sizes on the absorbance value of Qingzhuan tea extract}

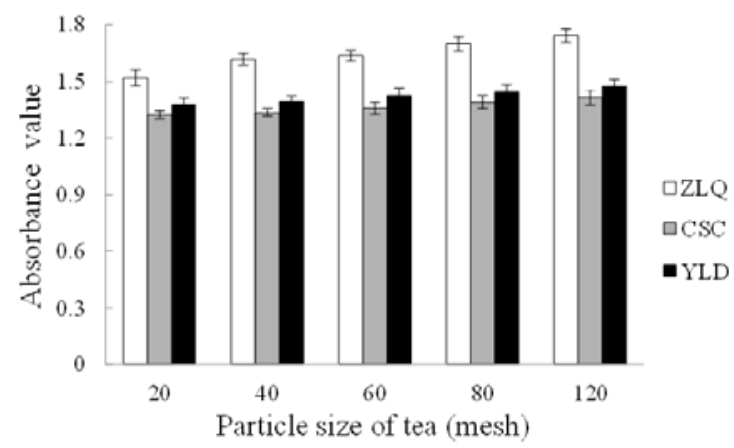

Fig.2 Influence of tea particle sizes on absorbance
As Fig. 2 showed the absorbance value of Qingzhuan tea aqueous extracts increased with the increase of tea particle size whether it was ZLQ, CSC or YLD. The result was consistent with Yang[8]: the smaller the tea particles were, the more the particles exposed to the solution, and the shorter the distance of substance from the inside of the tea leaf was, the faster the substance could be dissolved in water. In three types of Hubei Qingzhuan tea the absorbance of ZLQ tea was much higher than CSC and YLD when the tea were in the same size. It might due to ZLQ fermented longer, which needed further exploration.

\subsubsection{The effect of tea-water ratios on the absorbance value of Qingzhuan tea extract}

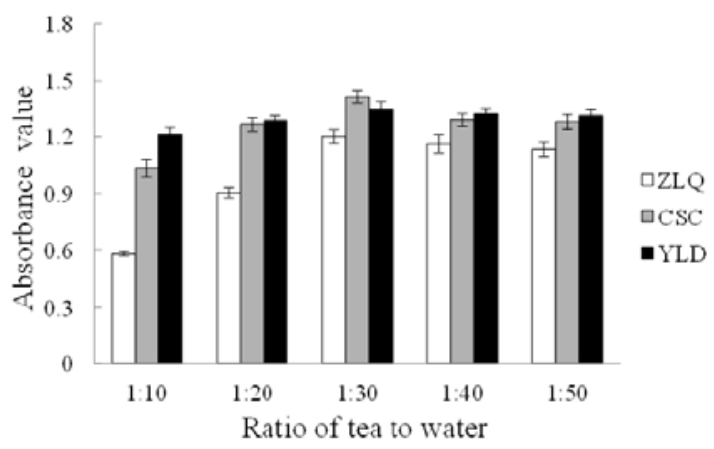

Fig.3 Influence of tea-water ratios on absorbance

In the single factor experiments of "tea-water ratio", equal-weight tea were extracted at different tea-water ratios. After the extraction, the absorbance value were not comparable for the different amount of water, so the supernatant must be diluted to constant volume. And then the higher absorbance value of the solution indicated the higher extraction efficiency.

With the increase of tea-water ratio, the absorbance values of different tea extracts increased first and then decreased (Fig.3). The extract concentration increased when the tea-water ratio changed from 1:10 to $1: 30$, and reached the peak value at the tea-water ratio of 1:30. When the water was further added, the extract concentration decreased instead. The result was consistent with Yang's study of production technology of Pu'er instant tea powder[9]. When the tea-water ratio was too low, the extract was not completely dissolved, and the limited amount of water restricted the extraction efficiency. As the tea-water ratio raised, the extract concentration gradually increased. When the tea-water ratio improved to some extend, the water was gradually excessive, which would induce the extraction efficiency decreased while the tea content was fully dissolved. So the tea-water ratio should be controlled in an appropriate range. In this experiment, when the tea-water ratio was $1: 30$, the extraction efficiency of Qingzhuan tea was the best. Lai'sstudy had shown that tea polyphenols, caffeine, free amino acids and three pigments (theaflavin, thearubin, theophyllin) content of the Qingzhuan tea were lower, but the soluble sugar content was higher comparing with Fuzhuan tea and Heizhuan tea[10] . 


\subsubsection{The effect of extracting temperatures on the absorbance value of Qingzhuan tea extract}

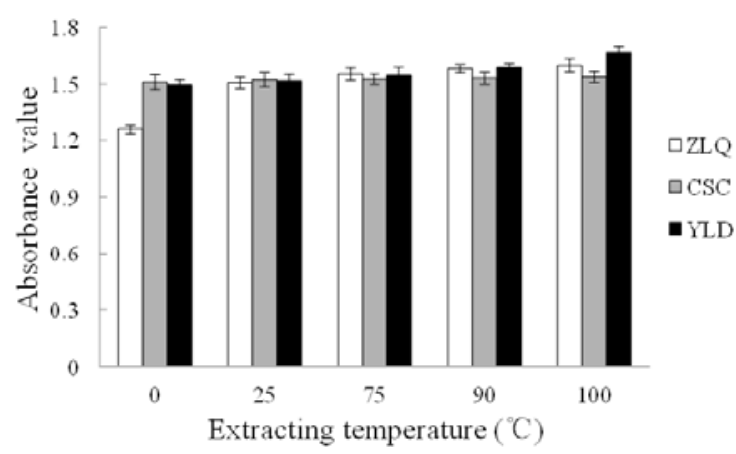

Fig.4 Influence of extracting temperatures on absorbance

With the increase of the extracting temperature, Qingzhuan tea brewed from "cold brewing" to "hot brewing". The absorbance values of the three types of tea were increased too (Fig.4), which was the same as Yang's findings[9]. The higher the temperature was, the faster the molecular diffusion rate was, which was beneficial to accelerate the extracting process. And the tea content was distributed in the deep layer of the mesophyll tissue, which had good stability and needed high temperature to extracted. There were increasing trends of tea polyphenols, amino acids, flavonoids, caffeine and other biochemical components in Wuyi Cinnamon soup with the increase of brewing temperature $\left(80^{\circ} \mathrm{C}, 90^{\circ} \mathrm{C}, 100^{\circ} \mathrm{C}\right)$ and the brewing time[11]. The brewing and extraction temperature not only affected the contents of the tea soup, but also influenced the color of the soup. In general, theaflavin and thearubin showed the maximum extracting amount at $80^{\circ} \mathrm{Cand} 90^{\circ} \mathrm{C}$ while the water temperature exceeded $90^{\circ} \mathrm{C}$, the thearubin would be converted into theaflavin, causing the tea soup darker and affecting the taste quality of the tea soup[12].

\subsubsection{The effect of extracting time on the absorbance of Qingzhuan tea extract}

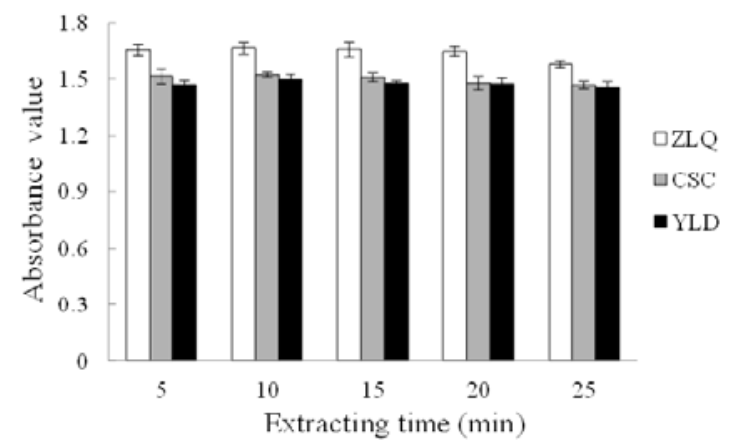

Fig.5 Influence of extracting time on absorbance

From the Fig.5, it could be seen that with the extension of the extracting time, the absorbance values of the three types of Qingzhuan tea all showed a trend of increasing first and then decreasing. The absorption of ZLQ reached its maximum value after 15 minutes of extracting, while CSC and YLD were 10 minutes. A study on the extracting rate of black teafound that the extracting time had a significant effect on the extracting rate of tea polyphenols, amino acids, and Aqueous extract[10]. The reason might be that the longer the extracting time was, the more the tea extracted. After a while, the system reached osmotic equilibrium, then extracting time had little influence on extraction efficiency. If continued to extract, the soup might be evaporated and oxidized.

Comparing with the three kinds of Qingzhuan tea, CSC and YLD performed consistently while the extraction efficiency of ZLQ was greatly affected by "particle size of tea" and "extracting time".

\subsection{Orthogonal experiment results of Qingzhuan instant tea powder production technology}

\subsubsection{Range analysis}

According to the results of single-factor experiments, "particle size of tea, tea-water ratio, extracting temperature, extracting time" this four factors and three levels was chosen in the orthogonal experiment. The experimental results were shown in Table 2. In the process of making instant tea powder of Qingzhuan tea, the ranges of particle size of tea, tea-water ratio, extracting temperature, extracting time were $0.240,0.070$ 0.253 and 0.031 respectively, which indicated that extracting temperature and particle size of tea had the greatest influence on the extraction efficiency of Qingzhuan tea, followed by the tea-water ratio, while the extracting time had little influence on the extraction efficiency.

According to the mean absorbance analysis of the four factors at different levels, the best technological conditions for the instant powder production of Qingzhuan tea are particle size of tea 120 mesh, the tea-water ratio of 1:40, and the extracting temperature of $100^{\circ} \mathrm{C}$.

The average yield of the three kinds of Hubei Qingzhuan tea was $14.3 \%$ after extraction and freeze-drying.

Table 2. Orthogonal experiment results of "technology of Qingzhuan tea instant powder"

\begin{tabular}{|c|c|c|c|c|c|}
\hline & A & B & C & D & Abs. \\
\hline Exp.1 & 60 & $1: 40$ & 75 & 5 & 1.133 \\
\hline Exp.2 & 80 & $1: 30$ & 75 & 10 & 1.145 \\
\hline Exp.3 & 120 & $1: 20$ & 75 & 15 & 1.347 \\
\hline Exp.4 & 80 & $1: 40$ & 90 & 15 & 1.333 \\
\hline Exp.5 & 120 & $1: 30$ & 90 & 5 & 1.486 \\
\hline Exp.6 & 60 & $1: 20$ & 90 & 10 & 1.228 \\
\hline Exp.7 & 120 & $1: 40$ & 100 & 10 & 1.649 \\
\hline Exp.8 & 60 & $1: 30$ & 100 & 15 & 1.363 \\
\hline Exp.9 & 80 & $1: 20$ & 100 & 5 & 1.331 \\
\hline Mean 1 & 1.241 & 1.372 & 1.208 & 1.317 & \\
\hline Mean 2 & 1.270 & 1.331 & 1.349 & 1.341 & \\
\hline Mean 3 & 1.494 & 1.302 & 1.448 & 1.348 & \\
\hline range & 0.253 & 0.070 & 0.240 & 0.031 & \\
\hline
\end{tabular}

Note: A: particle size of tea (mesh), B: tea-water ratio, C: extracting temperature $\left({ }^{\circ} \mathrm{C}\right)$, D: extracting time $(\mathrm{min})$. 


\subsubsection{Variance analysis}

In this experiment, the main effect of each factor was investigated without considering the interaction. The analysis of variance was shown in Table 3. According to variance analysis, "particle size of tea", "tea-water ratio" and "extracting temperature" had significant influence on extraction efficiency, while "extraction time" had little. This conclusion was consistent with the result of range analysis. As we all known, water temperature, brewing time and tea-water ratio were the three major elements of tea making. In this study "extraction time" had little influence maybe because the tea leaves were crushed in a tissue crusher and sieved, the granularity of tea leaves was relatively small, the contact area between tea leaves and water was large, and the substance permeation balance was easy to be achieved.

Table 3. Tests of between-subjects effects in "technology of Qingzhuan tea instant powder"

\begin{tabular}{|c|c|c|c|c|c|}
\hline $\begin{array}{c}\text { Source of } \\
\text { variation }\end{array}$ & $d f$ & SS & MS & $\mathrm{F}$ & $p$ \\
\hline A & 2 & 0.199 & 0.1 & 5.923 & 0.011 \\
\hline B & 2 & 0.218 & 0.109 & 6.464 & 0.008 \\
\hline C & 2 & 0.164 & 0.082 & 4.869 & 0.020 \\
\hline D & 2 & 0.051 & 0.025 & 1.501 & 0.249 \\
\hline Error & 18 & 0.303 & 0.017 & & \\
\hline Total & 27 & 49.052 & & & \\
\hline
\end{tabular}

Note: A: particle size of tea (mesh), B: tea-water ratio, C: extracting temperature $\left({ }^{\circ} \mathrm{C}\right)$, D: extracting time $(\min )$.

\section{Conclusion and prospect}

Although Chinese instant tea powder production ranks first in the world, instant dark tea powder is rare in the market. In this study, ultrasonic assisted extraction and freeze-drying technology were adopted to optimize the production process of Hubei Qingzhuan tea high fragrance cold solubility instant powder, using absorbance value as a detection index by single factor experiments combined with orthogonal experiment, which could provide a beneficial reference for deep utilization of Hubei Qingzhuan tea. The data showed that the particle size of tea, tea-water ratio and extracting temperature significantly affected the extraction efficiency while extracting time did little when ultrasonic was used to assist extraction. The extraction efficiency was the highest when using 120-mesh dark tea, adding water at the ratio of $1: 40$, and the extracting temperature of $100^{\circ} \mathrm{C}$. The average yield of the three kinds of Hubei Qingzhuan tea was $14.3 \%$ after extraction and freeze-drying. Comparing with the brewed Qingzhuan tea, the instant powder had good cold solubility and poor flavor. It was necessary to use "fragrance recovery technology" to improve the tea flavor. Furthermore, the freeze-drying technology could not be continuously produced, and the energy consumption was very high, so it needed further exploration.

\section{Acknowledgement}

We acknowledge the financial support from Scientific research project of Hubei Provincial Education Department (B2016369).

\section{References}

1. Li Huihui, Zhang Yun, Bi Shen, etc. Modern food, 9,11-13+16 (2018).

2. Wang Wei, Gao Xiaqi, Hu Jun, etc. Bachu Med.J. 2, 9-15 (2019).

3. Mao Rong, Zhang Jingling, Du Guang. J. Hubei Univ. Sci.Techn. (Med. Sci.), 33(01), 7-9 (2019).

4. Tang Fei, Zhang Yuefeng, Qu Fengfeng, etc. Hubei Agric. Sci.12,124-129 (2019).

5. Liu Zhonghua. J.Tea Sci. 39(02),115-122 (2019).

6. Zheng Pengcheng, Liu Panpan, Wang Shengpeng, etc. Sci Tech. Food Ind. 39(22),88-92 (2018).

7. Zhang Liang, Lv Yangjun, Duan Yuwei, etc. Chin Tea Process. 1,33-39 (2017).

8. Yang Liuyang, Jiang Heyuan, Zhang Jiangyong, etc. J. Food Safety Qua.Inspec.4,1193-8 (2015).

9. Yang Zhuan, Guo Guiyi, Wang Qiaojiang, etc. Sci. Tech. Food Ind. 37(21),243-248 (2016).

10. Lai Xingfei, Bai Zheng, Li Zhifang, etc. Sci.Techn.Food Ind. 33(8),374-376 (2012).

11. Wu Na, Gou Gaozhang, Xu Shijuan, etc. Food Res.Deve. 37(4),139-143 (2016).

12. Ma Jingyu, Liu Qiang, Sun Yun, etc. Chin.tea. 5, 21-24 (2019). 